\title{
Gallbladder volvulus
}

\author{
Laureline Moser ${ }^{1}$, Gaëtan-Romain Joliat ${ }^{2}$, Parissa Tabrizian ${ }^{3}$, Luca Di Mare ${ }^{1}$, David Petermann ${ }^{1}$, \\ Nermin Halkic ${ }^{2}$, Nicolas Demartines ${ }^{2}$, Ismail Labgaa ${ }^{1,2}$
}

${ }^{1}$ Department of General and Visceral Surgery, EHC Hospital, Morges, Switzerland; ${ }^{2}$ Department of Visceral Surgery, Lausanne University Hospital CHUV, Lausanne, Switzerland; ${ }^{3}$ Department of Liver Surgery, Recanati/Miller Transplantation Institute, Icahn School of Medicine at Mount Sinai, New York, NY, USA

Correspondence to: Nicolas Demartines, MD. Professor and Chairman, University Hospital of Lausanne (CHUV), Department of Visceral Surgery, Rue du Bugnon 46, CH-1011 Lausanne, Switzerland. Email: Demartines@chuv.ch.

Submitted Oct 23, 2020. Accepted for publication Dec 24, 2020.

doi: 10.21037/hbsn-20-771

View this article at: http://dx.doi.org/10.21037/hbsn-20-771

\section{Background}

Like colon or stomach, gallbladder can twist, leading to a volvulus; an entity first described by Wendel in 1898 (1). Gallbladder volvulus (GV) is a relatively rare disease occurring when the gallbladder rotates on its own axis, along the cystic duct (Figure 1). Different types of rotation have been described: incomplete $v s$. complete $\left(\leq 180^{\circ} v\right.$ s. $>180^{\circ}$ ) and clockwise $v s$. anticlockwise (2). As the rotation might involve the cystic artery, blood supply may be compromised, resulting in ischemia and eventually necrosis. GV constitutes an acute abdominal condition associated with poor outcomes, if not diagnosed and treated in time.

The exact cause of GV remains unknown although several mechanisms have been postulated. Those include anatomical/hereditary, mechanical, physiological and hormonal risk factors (Table S1) (3).

Various theories have been suggested to explain GV. By increasing the gallbladder weight, gallstones would increase the risk of $\mathrm{GV}(4,5)$, a questionable theory. Other authors hypothesized that GV may result from peristaltic movements; clockwise rotations being caused by the stomach and anticlockwise rotation by the transverse colon (2). This hypothesis is also debated. Another perspective relies on the physiological changes induced with ageing, including loss of visceral fat and fibrous connective tissue, which would allow a "freely hanging" gallbladder that increases the risk of GV (3). Overall, data suggest that $\mathrm{GV}$ is a multifactorial disease whose cases do not necessarily share the same etiology.

This review aimed to provide a comprehensive overview of the available data on GV in terms of epidemiology, clinical presentation, diagnosis, treatment and outcomes.

\section{Epidemiology}

The present review of the literature identified 178 articles, mostly case reports, including a total of 477 cases of GV. These publications are detailed in https://cdn.amegroups. $\mathrm{cn} /$ static/public/hbsn-20-771-1.xlsx and the strategy and selection criteria are detailed in Appendix 1. Characteristics of the patients are summarized in Table 1 .

A wide range of incidence rates have been reported. In Japan, GV rates reached up to $8.1 \%$ and $11.6 \%$ in autopsy and surgical series, respectively (4). Overall, GV represents around 1 case per 350,000 hospital admissions (6-8). Due to a difficult diagnosis, its incidence and significance are likely underestimated. Although a majority of cases were reported in Asia, its geographical distribution remains unclear.

Data on demographics are quite consistent. Although GV can occur at any age, two peaks of incidence have been described: a first minor one in pediatric patients ( $<18$ years) and a second predominant one in elderly patients ( $>70$ years). Mean age was 65 years. Gender appeared to have an opposite impact in these two groups of patients: boys are at increased risk to develop GV than girls in the pediatric subgroup with a 5:1 ratio, whereas GV is more prevalent in women within adult patients, with a 5:1 ratio. Overall, GV was diagnosed in 361 (76.2\%) women and 113 (23.8\%) men.

While hereditary factors are likely responsible for GV in pediatrics, physiological changes associated with ageing appeared determinant of GV in adults. Therefore, thin 
A

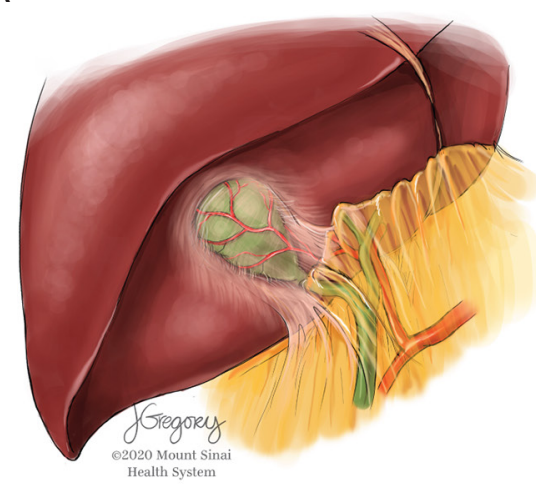

B

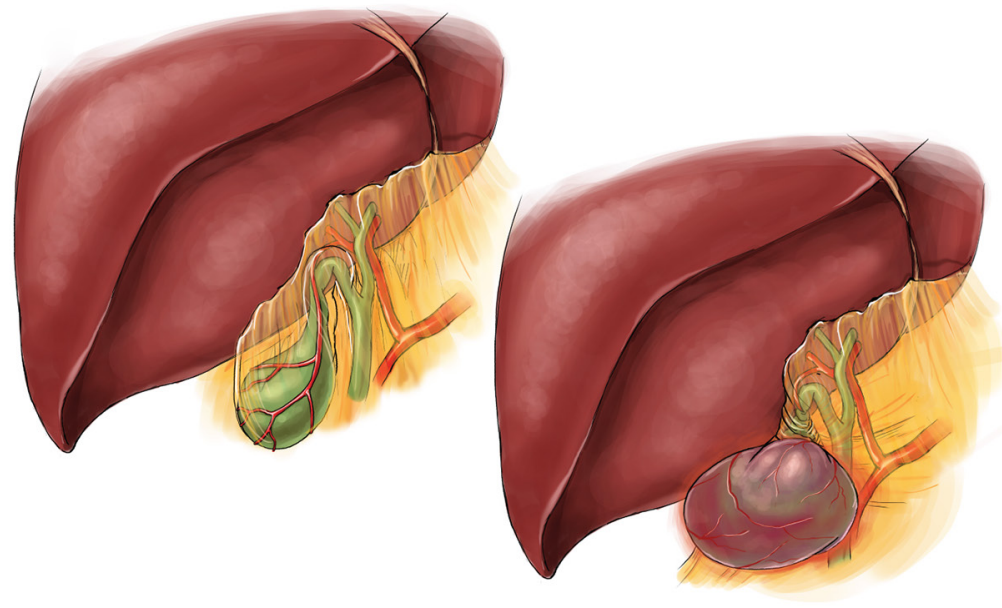

Figure 1 Gallbladder volvulus. (A) Normal gallbladder. (B) Anatomical variant of a gallbladder ("freely hanging") rotating on its own axis and thereby resulting in a gallbladder volvulus.

women at age of 70 years represent the archetype of patients who develop GV (9).

\section{Clinical presentation}

Both symptoms and signs are non-specific; alone, they do not permit to reliably diagnose GV. Of note, the clinical presentation strikingly mimics acute cholecystitis.

Abdominal pain (467/477, 98\%), vomiting (245/477, $51 \%)$, nausea $(201 / 477,42 \%)$ and fever $(131 / 477,28 \%)$ are the most common symptoms.

On physical examination, positive Murphy's sign and a palpable mass in the right upper quadrant might be observed. The latter is more specific but can be difficult to detect. Jaundice is however uncommon (2/477, 0.4\%).

\section{Diagnostic strategy}

Diagnosis was made pre-operatively in only 80 (17\%) patients.

The contribution of laboratory tests is poor. Elevated inflammatory markers such as C-reactive protein (CRP) and white blood cells count (WBC) are detected in 127/232 (55\%) cases. Conversely, and because the main bile duct is rarely obstructed in GV, liver tests are within normal range in about $85 \%$ of patients (197/232).

Imaging provides the main contribution for $\mathrm{GV}$ diagnosis. Even when technologies like magnetic resonance imaging (MRI) or computed tomography (CT) scanner are available, diagnosis of GV remains challenging and is preoperatively established only in a minority of cases. Ultrasound (US), CT and MRI were performed in 115/232 (50\%), 105/232 (45\%) and 17/232 (7\%) patients. They respectively allowed making diagnosis in 14/115 (12\%), $32 / 105$ (30\%) and 10/17 (59\%) cases.

Unfortunately, there is no available data on the performance of the different types of imaging techniques. Likewise, no study compared their performance.

Standard X-ray of the abdomen is not useful in GV. US allows detecting a gallbladder with an unusual location (outside its anatomical fossa), with thickened wall and eventually gallstones. Doppler-US color has the advantage of assessing the blood flow, determining whether the cystic artery is obstructed or not. CT-scanner and MRI both permit to detect the pathognomonic signs of GV; those include: (I) distended gallbladder, (II) pericholecystic fluid, (III) gallbladder outside its anatomical fossa, (IV) loss of enhancement, and (V) abrupt tapering of the cystic duct (Figure S1) $(4,5,10,11)$. Magnetic resonance cholangiopancreatography (MRCP) is certainly the radiological exam of choice for the diagnosis of $\mathrm{GV}$.

\section{Treatment}

As GV can easily be misdiagnosed as acalculous cholecystitis and typically occurs in frail elderly patients, the risk of misleadingly treat $\mathrm{GV}$ patients with antibiotics is increased. Such attempt, even with broad-spectrum antibiotics, is 
Table 1 Characteristics of patients with gallbladder volvulus

\begin{tabular}{|c|c|}
\hline Characteristics & $\mathrm{N}[\%]$ \\
\hline \multicolumn{2}{|l|}{ Demographics } \\
\hline Age (years)* & 64.8 \\
\hline Gender (female) & $361 / 474[76]$ \\
\hline \multicolumn{2}{|l|}{ Clinical presentation } \\
\hline Abdominal pain & $467 / 477[98]$ \\
\hline Nausea & $201 / 477$ [42] \\
\hline Vomiting & $245 / 477[51]$ \\
\hline Fever & $131 / 477[28]$ \\
\hline Jaundice & $2 / 477[0.4]$ \\
\hline \multicolumn{2}{|l|}{ Laboratory tests } \\
\hline Elevated WBC or CRP & $127 / 232[55]$ \\
\hline Elevated liver tests & $35 / 232[15]$ \\
\hline \multicolumn{2}{|l|}{ Imaging } \\
\hline US: performed/diagnostic & $115 / 232$ [50], 14/115 [12] \\
\hline CT: performed/diagnostic & 105/232 [45], 32/105 [30] \\
\hline MRI: performed/diagnostic & $17 / 232[7], 10 / 17$ [59] \\
\hline \multicolumn{2}{|l|}{ Treatment } \\
\hline Surgery/laparoscopy & 470/473 [99], 55/205 [27] \\
\hline \multicolumn{2}{|l|}{ Outcomes } \\
\hline Morbidity & $33 / 204[16]$ \\
\hline Mortality & $27 / 257[6]$ \\
\hline
\end{tabular}

*, mean age. WBC, white blood cells count; CRP, C-reactive protein; US, ultrasound; CT, computed tomography; MRI, magnetic resonance imaging.

likely to result in no-response to therapy and poor outcomes $(4,10,12)$.

The mechanical nature of GV necessitates to be mechanically treated. Therapeutic options include endoscopy with endoscopic retrograde cholangiopancreatography (ERCP) and surgery. Successful endoscopic treatment of $G V$ is however anecdotic, with only three reports of gallbladder detorsion by ERCP (13). Due to the common minimal fixation of the gallbladder to liver bed in $\mathrm{GV}$ cases, percutaneous transhepatic cholecyst drainage should be avoided as it may induce bile leakage and biliary peritonitis. Surgery constitutes the treatment of choice in GV management. In this review, 470/473 (99\%) patients underwent surgery with $55 / 205$ (27\%) cases reporting laparoscopic approach. These data raise two comments: the high proportion of unavailable data for the surgical approach and the low rate of laparoscopy. The first case of laparoscopic cholecystectomy for GV was reported in 1993, by Desai et al. (13). Meanwhile, 55 and 71 cases treated with laparoscopic and open cholecystectomy were reported, respectively. As expected, these detailed results show an increase in the rate of laparoscopic cholecystectomy for GV overtime.

Like any other indications of cholecystectomy, it can be either performed by laparotomy or laparoscopy. The technique is feasible, safe and can be performed for any types of GV. There is no available data comparing postoperative outcomes of laparotomy vs. laparoscopy. Nonetheless, the evidence on cholecystectomy for other causes than GV can be reasonably extrapolate, which demonstrated benefits associated with laparoscopy: less pain, lower rate of postoperative complications and shorter length of stay $(\mathrm{LoS})(14,15)$. Laparoscopy should also be the first approach for $\mathrm{GV}$, whenever possible.

\section{Outcomes}

Undiagnosed GV leads to the progression of the disease, encompassing ischemia, necrosis, perforation and eventually multi-organ failure. Overall, morbidity and mortality were reported in 33/204 (16\%) and 27/457 (6\%), respectively. Of note, among the 27 cases of death, 6 patients had a CTscanner but none underwent MRI.

Besides usual prognostic factors such as age and comorbidities, the delay between onset of symptoms and surgery is a prognostic factor. A delay $>2$ days was associated with an increased mortality (8). Although these data are not recent, the association between an increased preoperative delay and worse outcomes seems reasonable.

\section{Discussion}

The level of evidence on GV is solely based on case reports and case series. Future studies including prospective cohorts or autopsy research would be valuable to reduce the selection bias. With 477 included patients, the present systematic review is the largest one on the topic. In 1999, Nakao et al. reported a large series with 246 cases of GV (4), which only included 3 cases reported in English publications; the other cases were retrieved from a national Japanese database and therefore cannot necessarily be extrapolated to Western countries. Later, Reilly et al. 
published the only other available systematic review with 324 cases of GV (5). An important limitation of their review is the lack of details and transparency regarding the included cases, with discrepancy between the list of publications presumably selected $(n=521)$ and the total number of GV retrieved cases $(n=324)$, which makes the results hardly interpretable. In addition of providing detailed and trackable data, the present studies integrates a total of 153 new cases which were not included in their analyses.

GV is certainly an underestimated and underdiagnosed disease that can implicate clinicians from various specialties: gastroenterology, surgery, emergency, intensive care and internal medicine.

\section{Conclusions}

$\mathrm{GV}$ is a relatively rare condition whose diagnosis is difficult. It should be integrated in the differential diagnosis of cholecystitis, particularly in elderly thin women and in patients with no response to antibiotics for suspected cholecystitis. Surgery is the treatment of choice in GV management; it should be performed as early as possible, by laparoscopy, since delayed cholecystectomy is associated with poor outcomes including mortality.

\section{Acknowledgments}

The authors would like to thank Jill Gregory for the design of Figure 1, as well as the Medical Library of the Research and Education Department at Lausanne University Hospital (CHUV), particularly Mrs. Cécile Jaques for her assistance in the review of the literature.

Funding: None.

\section{Footnote}

Provenance and Peer Review: This article was a standard submission to the editorial office of Hepatobiliary Surgery and Nutrition. The article has undergone external peer review.

Conflicts of Interest: All authors have completed the ICMJE uniform disclosure form (available at https://hbsn. amegroups.com/article/view/10.21037/hbsn-20-771/coif). The authors have no conflicts of interest to declare.

Ethical Statement: The authors are accountable for all aspects of the work in ensuring that questions related to the accuracy or integrity of any part of the work are appropriately investigated and resolved.

Open Access Statement: This is an Open Access article distributed in accordance with the Creative Commons Attribution-NonCommercial-NoDerivs 4.0 International License (CC BY-NC-ND 4.0), which permits the noncommercial replication and distribution of the article with the strict proviso that no changes or edits are made and the original work is properly cited (including links to both the formal publication through the relevant DOI and the license). See: https://creativecommons.org/licenses/by-nc-nd/4.0/.

\section{References}

1. Wendel AV. VI. A Case of Floating Gall-Bladder and Kidney complicated by Cholelithiasis, with Perforation of the Gall-Bladder. Ann Surg 1898;27:199-202.

2. Kachi A, Nicolas G, Nasser J, et al. A Rare Presentation of Gall Bladder Volvulus: A Case Report. Am J Case Rep 2019;20:1466-70.

3. Shaikh AA, Charles A, Domingo S, et al. Gallbladder volvulus: report of two original cases and review of the literature. Am Surg 2005;71:87-9.

4. Nakao A, Matsuda T, Funabiki S, et al. Gallbladder torsion: case report and review of 245 cases reported in the Japanese literature. J Hepatobiliary Pancreat Surg 1999;6:418-21.

5. Reilly DJ, Kalogeropoulos G, Thiruchelvam D. Torsion of the gallbladder: a systematic review. HPB (Oxford) 2012;14:669-72.

6. Farhat W, Mabrouk MB, Ammar H, et al. Gallbladder volvulus: A case report and review of the literature. Int J Surg Case Rep 2019;60:75-8.

7. Pottorf BJ, Alfaro L, Hollis HW. A Clinician's Guide to the Diagnosis and Management of Gallbladder Volvulus. Perm J 2013;17:80-3.

8. Yeh HC, Weiss MF, Gerson CD. Torsion of the gallbladder: the ultrasonographic features. J Clin Ultrasound 1989;17:123-5.

9. Moser L, Labgaa I, Di Mare L. An Uncommon Cause of Cholecystitis. Gastroenterology 2020;158:e10-1.

10. Safadi RR, Abu-Yousef MM, Farah AS, et al. Preoperative sonographic diagnosis of gallbladder torsion: report of two cases. J Ultrasound Med 1993;12:296-8.

11. Merine D, Meziane M, Fishman EK. CT diagnosis of gallbladder torsion. J Comput Assist Tomogr 
1987;11:712-3.

12. Schilling JU, Wehrmann U, Saeger HD. Emergent cholecystectomy after acute cholecystitis with no improvement from i.v. antibiotics. Chirurg 2005;76:1082-5.

13. Desai HP, Gold BM, Burakoff R. Intermittent positional torsion of the gallbladder demonstrated on ERCP. Gastrointest Endosc 1993;39:572-3.

14. Keus F, Gooszen HG, Van Laarhoven CJ. Systematic

Cite this article as: Moser L, Joliat GR, Tabrizian P, Di Mare L, Petermann D, Halkic N, Demartines N, Labgaa I. Gallbladder volvulus. HepatoBiliary Surg Nutr 2021;10(2):249-253. doi: 10.21037/hbsn-20-771 review: open, small-incision or laparoscopic cholecystectomy for symptomatic cholecystolithiasis. Aliment Pharmacol Ther 2009;29:359-78.

15. Tan X, Wang G, Tang Y, et al. Minilaparoscopic versus single incision cholecystectomy for the treatment of cholecystolithiasis: a meta-analysis and systematic review. BMC Surg 2017;17:91. 

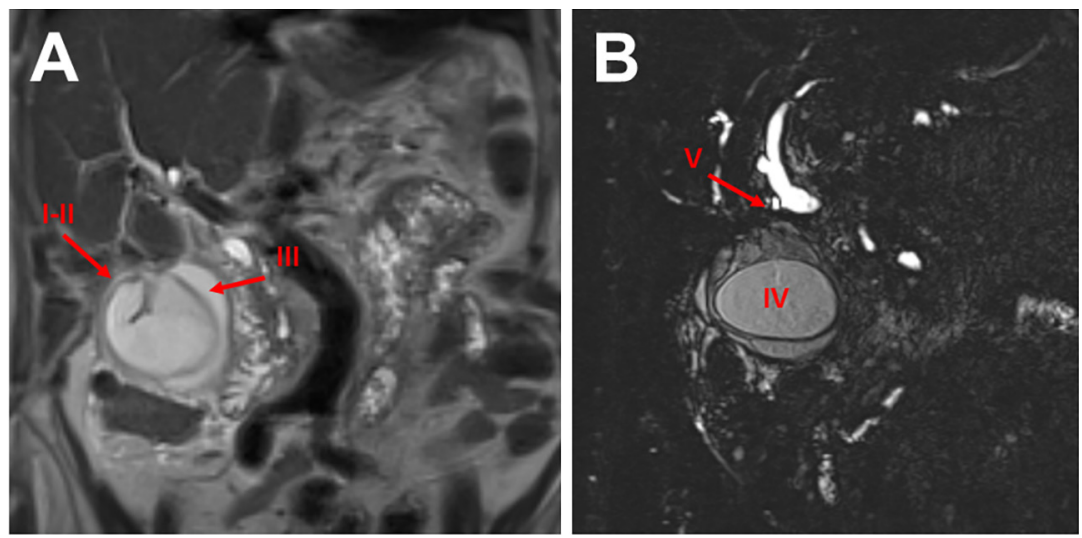

Figure S1 Radiological features of gallbladder volvulus. (A) Magnetic resonance cholangiopancreatography (coronal axis) showing (I) a distended gallbladder, (II) gallbladder outside its anatomical fossa and (III) pericholecystic fluid. (B) Magnetic resonance cholangiopancreatography (coronal axis) showing (IV) loss of enhancement and (V) abrupt tapering of the cystic duct.

Table S1 Risk factors of gallbladder volvulus

Risk factors

Anatomical/hereditary

- Variant cystic mesentery (absent, or abnormally short/long)

- Tortuous and/or arteriosclerotic cystic artery

- Pathological spine (kyphosis, scoliosis)

- Free hepatic lobe

- Lack of coronary and/or triangular ligament(s)

Mechanical

- Sudden changes in body position

- Abdominal trauma

- Peristalsis (stomach, duodenum and colon)

Physiological

- Weight loss

- Loss of fibrous connective tissue (ageing)

Hormonal

- $\uparrow$ Cholecystokinin 


\section{Appendix 1}

\section{Strategy and selection criteria}

The review of the literature was conducted via PubMed, using the following MeSH terms and keywords: "volvulus" AND "galbblader", "volvulus" AND "gall-bladder", "torsion" AND "gallbladder" or "torsion" AND "gall-bladder". Search was limited to full-text articles published in English, French or German until January 4th 2020. Cross-referencing of the bibliographies from the eligible articles was also performed. All patients with gallbladder volvulus were included. No exclusion criteria were applied. 